\title{
ORIGINAL ARTICLE \\ Complementary sex determination, inbreeding depression and inbreeding avoidance in a gregarious sawfly
}

\begin{abstract}
KE Harper ${ }^{1}$, RK Bagley ${ }^{1}$, KL Thompson ${ }^{2}$ and CR Linnen ${ }^{1}$
Although most Hymenoptera reproduce via arrhenotokous haplodiploidy, the underlying genetic mechanisms vary. Of these, the most widespread mechanism appears to be single-locus complementary sex determination (sI-CSD), in which individuals that are diploid and heterozygous at a sex-determining locus are female, and individuals that are homozygous or hemizygous are male. Because inbreeding increases the probability of producing diploid males, which are often sterile or inviable, sl-CSD can generate substantial inbreeding depression. To counteract this, Hymenoptera with traits that promote inbreeding, such as gregariousness, may evolve one or more of the following: inbreeding avoidance, functional diploid males or alternative sex determination mechanisms. Here, we investigate sex determination, inbreeding depression and inbreeding avoidance in Neodiprion lecontei, a gregarious, pine-feeding sawfly in the family Diprionidae. First, via inbreeding experiments and flow cytometry, we demonstrate that this species has CSD. By modeling expected sex ratios under different conditions, we also show that our data are consistent with sI-CSD. Second, via tracking survival in inbred and outbred families, we demonstrate that inbred families have reduced larval survival and that this mortality is partly attributable to the death of diploid males. Third, using a no-choice mating assay, we demonstrate that females are less willing to mate with siblings than nonsiblings. Together, these results suggest that inbreeding depression stemming from CSD has shaped mating behavior in $N$. lecontei. These results also set the stage for future comparative work that will investigate the interplay between sex determination, ecology and behavior in additional diprionid species that vary in larval gregariousness.
\end{abstract}

Heredity (2016) 117, 326-335; doi:10.1038/hdy.2016.46; published online 6 July 2016

\section{INTRODUCTION}

One of the four hyperdiverse insect clades, the order Hymenoptera contains over 115000 described species that collectively represent tremendous ecological, behavioral and morphological diversity. Research spanning the past eight decades has revealed that these insects also harbor striking variation in sex determination mechanisms (Snell, 1935; Whiting, 1943; Crozier, 1971; Cook, 1993a; Beukeboom, 1995; van Wilgenburg et al., 2006; Heimpel and de Boer, 2008). Although all hymenopterans are haplodiploid and most reproduce via arrhenotokous parthenogenesis, in which diploid females develop from fertilized eggs and haploid males develop from unfertilized eggs, some taxa have evolved other types of haplodiploidy. For example, both paternal genome elimination, in which males develop from fertilized eggs that have lost their paternal chromosome complement, and thelotoky, in which females lay unfertilized eggs that develop into diploid daughters, have been documented in the Hymenoptera. Moreover, even among species reproducing via arrhenotoky, there is variation in the molecular mechanisms of sex determination (Cook, 1993a; Heimpel and de Boer, 2008).

To date, several models have been proposed for sex determination under arrhenotoky. First, under complementary sex determination (CSD), individuals that are heterozygous at one or more sexdetermining loci develop into diploid females, whereas individuals homozygous (diploid) or hemizygous (haploid) at these loci develop into males. There are two types of CSD: single-locus CSD (sl-CSD), in which a single locus determines sex (Whiting, 1943), and multi-locus CSD (ml-CSD), in which multiple loci determine sex (Snell, 1935; Crozier, 1971; de Boer et al., 2008). A second model of sex determination in the Hymenoptera is genomic imprinting sex determination, in which female development requires the presence of a paternally derived, nonimprinted sex determination gene in addition to a maternally derived, imprinted copy (Poirie et al., 1992; Beukeboom, 1995; Beukeboom et al., 2007; Verhulst et al., 2010; van de Zande and Verhulst, 2014). Finally, the genic balance sex determination model posits that sex is determined by a combination of dosage-dependent 'femaleness' genes and dosage-independent 'maleness' genes (Cunha and Kerr, 1957). Of these models, only sl-CSD, ml-CSD and genomic imprinting sex determination have received empirical support, and sl-CSD appears to be the most widespread in the Hymenoptera (van Wilgenburg et al., 2006; Heimpel and de Boer, 2008; Asplen et al., 2009).

The interplay between sex determination mode and haplodiploidy can have important consequences for the intensity of inbreeding depression experienced by different hymenopteran taxa. Relative to diploid taxa, minimal inbreeding depression is expected under haplodiploidy because deleterious recessive alleles are purged every generation via haploid males (Bruckner, 1978; Henter, 2003). In support of this prediction, a meta-analysis revealed that haplodiploid hymenopterans and mites experience less inbreeding depression than diploid taxa (Henter, 2003). This does not imply, however, that

${ }^{1}$ Department of Biology, University of Kentucky, Lexington, KY, USA and ${ }^{2}$ Department of Statistics, University of Kentucky, Lexington, KY, USA Correspondence: Dr CR Linnen, Department of Biology, University of Kentucky, 204E Thomas Hunt Morgan Building, Lexington, KY 40506, USA E-mail: catherine.linnen@uky.edu

Received 4 December 2015; revised 23 March 2016; accepted 18 May 2016; published online 6 July 2016 
haplodiploid taxa are immune to inbreeding depression. For example, loci underlying female-limited traits and loci with overdominance (that is, heterozygotes are most fit) should still be subject to inbreeding depression (Henter, 2003). Moreover, for taxa with CSD, the production of diploid males, which are often inviable or sterile (Cook and Crozier, 1995; Heimpel and de Boer, 2008; Harpur et al., 2013), represents another source of inbreeding depression. Because the probability of diploid male production (hereafter, DMP) is much higher when there are fewer sex-determining loci, inbreeding depression stemming from DMP is expected to be most severe in taxa with sl-CSD.

Given the potentially large fitness cost of DMP, selection is expected to favor inbreeding avoidance mechanisms in taxa with sl-CSD. Multiple inbreeding avoidance strategies are possible, including temporal or spatial segregation of the sexes (Lee et al., 1998), premating refractory periods (Ode et al., 1995) and behavioral avoidance of kin mating (Ode et al., 1995; Metzger et al., 2010). Alternatively, in taxa in which ecological constraints make it difficult or impossible to avoid inbreeding, selection may favor an increase in the viability and fertility of diploid males (Cowan and Stahlhut, 2004; Elias et al., 2009) or the evolution of alternative sex determination strategies such as ml-CSD or genomic imprinting sex determination (Cook, 1993b; Dobson and Tanouye, 1998; Schrempf et al., 2006; van Wilgenburg et al., 2006). More generally, we can make the prediction that hymenopteran taxa with life histories that facilitate inbreeding will have one or more of the following: increased inbreeding avoidance, decreased inbreeding depression or alternative sex determination mechanisms (Henter, 2003; de Boer et al., 2007).

One factor that may affect the probability of inbreeding is the spatial distribution of siblings. In particular, it has been argued that compared with solitary species, gregarious species should have a higher incidence of sib-mating (Godfray, 1994; van Wilgenburg et al., 2006; de Boer et al., 2007). Consistent with the prediction that increased inbreeding should favor mechanisms that mitigate or eliminate DMP, CSD has been shown to be absent in two gregarious species of Cotesia (Niyibigira et al., 2004). Moreover, although sl-CSD has been confirmed in the gregarious parasitoid Cotesia glomerata (Zhou et al., 2006) and this species appears to lack kin-based mate preferences (Ruf et al., 2010), diploid C. glomerata males are viable and fertile (Elias et al., 2009). Collectively, work in Cotesia demonstrates the utility of examining closely related species for exploring the causes and consequences of variation in sex-determining mechanisms.

In this paper, we investigate sex determination, inbreeding depression and inbreeding avoidance in Neodiprion lecontei, a gregarious pine-feeding sawfly in the family Diprionidae (suborder: Symphyta; superfamily: Tenthredinoidea). Like Cotesia, diprionid sawflies vary in ecology and sex determination and are therefore potentially valuable models for exploring the interplay between these characteristics. To date, empirical work supports two modes of sex determination in the Diprionidae: thelotoky in Gilpinia hercyniae (=Diprion polytonum) (Smith, 1941) and sl-CSD in one Neodiprion species (N. nigroscutum) (Wallace and Smith, 1971). Sl-CSD has also been proposed for N. pinetum (as a personal communication in Stouthamer et al., 1992), but the supporting data have not been published. Diprionid species also exhibit variation in female oviposition behavior and larval gregariousness. For example, whereas N. nigroscutum females tend to distribute their eggs across multiple trees (Becker and Benjamin, 1967), N. lecontei females tend to lay their entire complement of eggs on a single branch terminus and gregarious larvae remain clustered in family groups throughout much of their development (Benjamin, 1955; Wilson et al., 1992). These behavioral differences should make inbreeding more likely in N. lecontei than in $N$. nigroscutum and other solitary diprionids, therefore increasing selection to reduce the impact of sl-CSD (or other sources of inbreeding depression) through one or more mechanisms. To test this prediction, we first assessed sex determination mechanism in $N$. lecontei via a combination of inbreeding experiments and flow cytometry. Next, we assessed inbreeding depression by comparing developmental mortality and cocoon weight between inbred and outbred families. Finally, to evaluate inbreeding avoidance, we conducted a series of no-choice mating assays. Collectively, these results demonstrate that $N$. lecontei has CSD, experiences inbreeding depression and exhibits behavioral inbreeding avoidance.

\section{MATERIALS AND METHODS}

\section{Insect collection and rearing}

To assess sex determination mechanism, inbreeding depression and inbreeding avoidance, we took advantage of rearing data, mating assays and frozen samples (for flow cytometry) collected for $N$. lecontei over a 3-year period. In total, 12 Neodiprion lines contributed to these analyses (Supplementary Table S1). Each line was established from field-collected larvae that were transported to the lab in paper bags and reared to adulthood to produce ' $\mathrm{G} 0$ ' adults that we then used to propagate additional generations (G1-G4) en masse. From these propagation cages, we also set up pairs of males and females to enable us to track fitness, mating behavior and DMP for individual families. All rearing methods are described in detail in the Supplementary Methods.

\section{Assessment of CSD}

CSD can be distinguished from alternative sex determination models via inbreeding experiments. Because inbreeding increases homozygosity, inbreeding under CSD is expected to result in the production of diploid males at the expense of diploid females, thereby increasing the sex ratio (proportion males). However, diploid male production alone is not definitive proof of CSD because these can arise via other mechanisms, such as genomic imprinting sex determination (Dobson and Tanouye, 1998). In contrast, inbreeding is not expected to affect sex ratio under noncomplementary mechanisms.

To test the CSD prediction that inbreeding will result in the production of diploid males, we used flow cytometry to examine the ploidy of males derived from mated sibling pairs. These sibling pairs were derived from a population collected in Lexington, KY (RB017; Supplementary Table S1). Briefly, pairs of adults were taken from the G2 propagation stock to establish sets of individuals of known relationship ('I' families), then propagated via mating brothers (that were found to be haploid, see Results) and sisters for an additional generation. A random subset of males from each generation had been frozen at $-80^{\circ} \mathrm{C}$. We analyzed all available frozen males from one I-G0 family $(n=11)$ and three I-G1 families $(n=6,8$ and 26).

To determine ploidy of males, we performed flow cytometry of propidium iodide (PI)-stained nuclei following the protocol in Hare and Johnston (2011), described in more detail in the Supplementary Methods. We analyzed stained samples on a BD FACSCalibur flow cytometer (BD Biosciences, San Jose, CA, USA) and called 2C peaks using Modfit LT software (Verity Software House, Topsham, ME, USA). In each flow cytometry run, we included as external standards a haploid male derived from a virgin female from the RB017 line and a G0 diploid female from a separate population (RB027, Supplementary Table S1). We then estimated relative genome size (RGS) for each unknown sample via comparison with haploid and diploid standards using the following

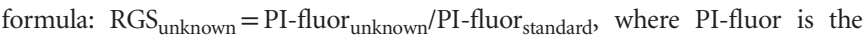
mean fluorescence of the $2 \mathrm{C}$ peaks for the samples and standards. Haploid samples are expected to have RGS values of 0.5 (compared with the diploid female standard) and 1.0 (compared with the haploid male standard); diploid samples are expected to have RGS values of 1.0 and 2.0.

We tested the CSD prediction that inbreeding will increase the sex ratio (proportion males) in two ways. First, we compared offspring sex ratio in inbred and outbred families. These families were established from G1 females from Lexington, KY (RB126) paired with either G1 males from the same population (RB126) or G2 males from Grayling, MI, USA (RB093; 
Supplementary Table S1). RB126 was founded from 9 females and 10 males reared from a single, isolated larval colony. Because $N$. lecontei females tend to lay their entire egg complement on a single branch terminus (Benjamin, 1955), the founding members of RB126 were most likely siblings. In total, we set up 18 inbred pairs $(\mathrm{RB} 126 \times \mathrm{RB} 126)$ and 21 outbred pairs $(\mathrm{RB} 126 \times \mathrm{RB} 093)$. For each pair, we tracked mating behavior to assess inbreeding avoidance as described below. Of these pairs, 11 inbred and 14 outbred pairs produced eggs; we used these families to track inbreeding depression as described below. To assess sex ratio, we used the 8 inbred and 13 outbred families that produced cocoons and adults. To determine the number of male and female cocoons in each family (cocoon size is sexually dimorphic; Wilson et al., 1992), we weighed all cocoons on a Mettler-Toledo (Columbus, OH, USA) balance. We then calculated sex ratio (proportion males) for each family using both cocoon data (number of male cocoons/total number of cocoons) and adult data (number of emerged males/total number of emerged adults). To compare sex ratios between inbred and outbred families, we used one-tailed $t$-tests. Sex ratios were transformed before analysis by taking the arcsine of the square root of the proportion males. As an alternative approach to analyzing the sex ratio data, we ran a log-binomial regression on the untransformed data using generalized estimating equations to account for observations clustered within families.

Under CSD, only matched pairs are expected to produce diploid males and male-biased sex ratios. To distinguish between matched and unmatched families in our inbred crosses, we compared Clopper-Pearson (exact) $95 \%$ confidence intervals for the observed sex ratio of each family (determined from cocoons rather than emerged adults to increase sample size) to sex ratios expected under an unmatched mating that we estimated using the observed sex ratios for outbred families. These and all other statistical analyses were performed in SAS Version 9.3 (SAS Institute Inc., Cary, NC, USA).

Second, we also examined how sex ratio changed over multiple generations of inbreeding in the lab. To do so, we compiled adult emergence data from nine different lab lines that were reared en masse for three-five generations (Supplementary Table S1). These lab colonies were founded from an average of 23 females (range: 6-72) and 23 males (range: 6-65). For each line, we calculated the sex ratio (number of emerged males/total number emerged adults) for each generation. To examine the impact of inbreeding on sex ratio, we used a repeated measures analysis of variance followed by post hoc pairwise comparisons. Before analysis, we took the arcsine of the square root of the proportion of males to put the variable on a continuous scale. We also analyzed the untransformed sex ratio data using a generalized linear model with binomial error structure, accounting for observations clustered within lines and repeated measures across generations, followed by post hoc pairwise comparisons.

\section{Assessment of inbreeding depression}

To determine whether inbred families had reduced fitness compared with outbred families, we tracked survival in 14 outbred $(\mathrm{RB} 126 \times \mathrm{RB} 093)$ and 11 inbred (RB126 $\times$ RB126) families (that is, the same families that were used for the sex ratio data). For each family, we recorded the number of eggs laid, larvae hatched, cocoons spun and adults enclosed. From these data, we calculated stage-specific survival rates (egg to larva; larva to cocoon; cocoon to adult). Because we were unable to confirm whether cocoons that failed to produce adults were dead or diapausing, we estimated overall survival from egg to cocoon rather than egg to adult. To compare numbers of progeny and survival rates between inbred and outbred families, we used one-tailed $t$-tests. To compare the numbers of males and females produced by inbred and outbred families, we used a mixed model as there were two observations (number of males and number of females) from each family. The model included sex, family type (inbred vs outbred) and the interaction as fixed effects and family as a random effect. All data were transformed before analysis (square root for progeny numbers; arcsine of the square root for survival rates).

Finally, in addition to survival and progeny number, we also examined offspring fecundity in inbred and outbred families. Because $N$. lecontei females emerge with their full complement of mature eggs, female weight is generally a good predictor of female fecundity (Benjamin, 1955). To verify this, we weighed 15 females from the RB017 line and then dissected them to obtain an egg count. To compare cocoon weights between inbred and outbred families we used a mixed model with family included as a random effect. Because cocoon sex was inferred on the basis of weight, male and female cocoon weights were analyzed separately to avoid circularity.

\section{Assessment of inbreeding avoidance}

To assess behavioral inbreeding avoidance, we conducted two sets of no-choice mating assays. The first set involved the inbred and outbred crosses described above $(\mathrm{RB} 126 \times \mathrm{RB} 126 \quad(n=18) ; \mathrm{RB} 126 \times \mathrm{RB} 093 \quad(n=21))$. Although we suspect that the RB126 line was initiated with siblings, and thus all individuals are expected to be closely related, the individuals used in these crosses were derived from a propagation line and the exact relationship of male/female pairs was unknown. We also suspect that some diploid males may have been present in the propagation line (see Results and Discussion) that may also affect mating behavior (Wallace and Smith, 1971). To address these issues, we set up a second set of mating assays. To ensure we were starting with a genetically diverse sample and to produce individuals of known sibling status, we paired G1 adults from two populations collected $130 \mathrm{~km}$ apart in Michigan (RB244 and RB261, both of which were established from $\geqslant 5$ distinct colonies; Supplementary Table S1). These pairings produced 25 families from which we sampled $n=36$ sibling pairs (same family) and $n=36$ nonsibling pairs (different families).

For all assays, we chose a no-choice mating design because it most closely approximates mating conditions in the wild (Benjamin, 1955; Wilson et al., 1992). Each female was paired with either a related or an unrelated male. In addition, only virgins were used and no individual was used in more than one assay. Each pair was placed in a new, empty $60 \mathrm{~mm} \times 15 \mathrm{~mm}$ petri dish and recorded for $75 \mathrm{~min}$ on either a Logitech or Microsoft webcam connected to a Lenovo Ideapad laptop. Each video consisted of three sibling pairs and three nonsibling pairs with position randomized to control for possible positional effects. All recordings took place in a controlled environment $\left(22^{\circ} \mathrm{C}, 70 \%\right.$ humidity). Videos were reviewed on a Macintosh computer using VLC media player (VideoLAN, Paris, France), and for each pair, we recorded whether mating occurred and, if so, the latency time to mating.

Within each set of crosses, we examined the impact of relative/sibling status on the mating outcome (presence/absence) using one-tailed Fisher's exact tests. Mating latencies were log-transformed, and then inbred and outbred matings were compared via one-tailed $t$-tests.

\section{RESULTS}

\section{Assessment of CSD}

Ploidy analysis in families derived from mated siblings confirmed that diploid males are produced in N. lecontei (Figure 1). Ploidy and sex ratio data from the I-G0 family indicate that the founding pair was not matched. In particular, this family had a female-biased sex ratio $(36 \%$ male) and $100 \%$ of the males tested $(n=11)$ were haploid. In contrast, observation of diploid males in each of the three sets of I-G1 offspring (observed fraction of diploid males (number of diploid males/ number tested) for each family: I-G1_3=18/26; I-G1_4=3/6; I-G1_5=1/8) indicates that all three families were derived from matched sibling pairs.

To assess the probability of observing three matched sibling pairs (inferred from the observation of at least one diploid male offspring) out of the three tested families, we performed exact binomial tests. We used these tests to evaluate three scenarios: the mother of the mated siblings had a single heterozygous sex-determining locus and did not share any alleles with the father; the mother had two heterozygous loci and one shared allele; and the mother had two heterozygous loci with no shared alleles. For these three scenarios, the probability of 'success' (observing a diploid male) for each 'trial' (family) was assumed to be $0.5,0.375$ and 0.25 , respectively. We found that our observed number of matched families was unlikely under models in which there were two or more heterozygous sex-determining loci in the mother (two loci, neither shared: $P=0.016$; two loci, one shared $P=0.053$; one unshared locus: $P=0.13$ ). These data are consistent with either 

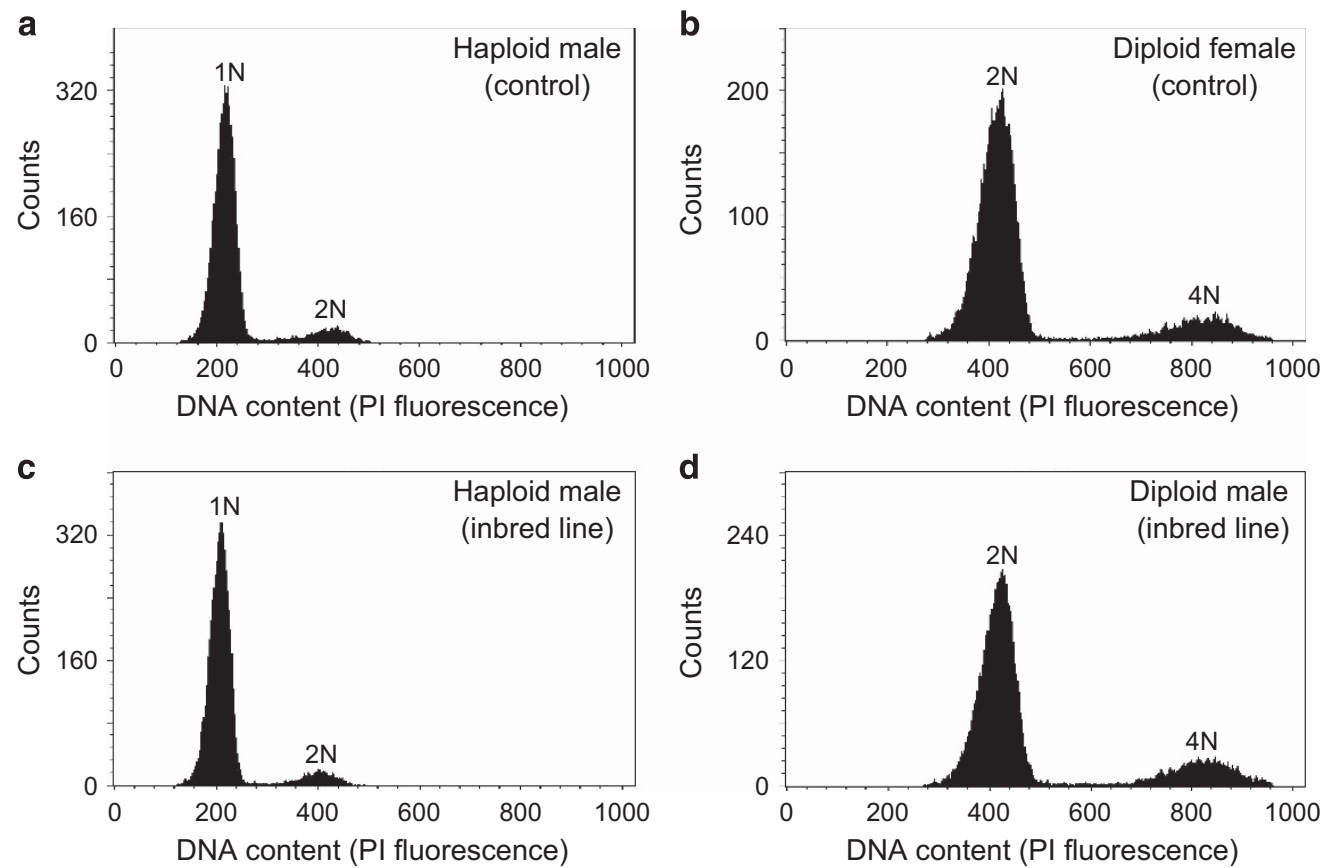

Figure 1 Representative flow cytometry results for (a) a haploid male control, (b) a diploid female control, (c) a haploid male and (d) a diploid male. Ploidy results for all unknowns were unambiguous. These experiments demonstrate that $N$. lecontei produces diploid males under inbreeding.
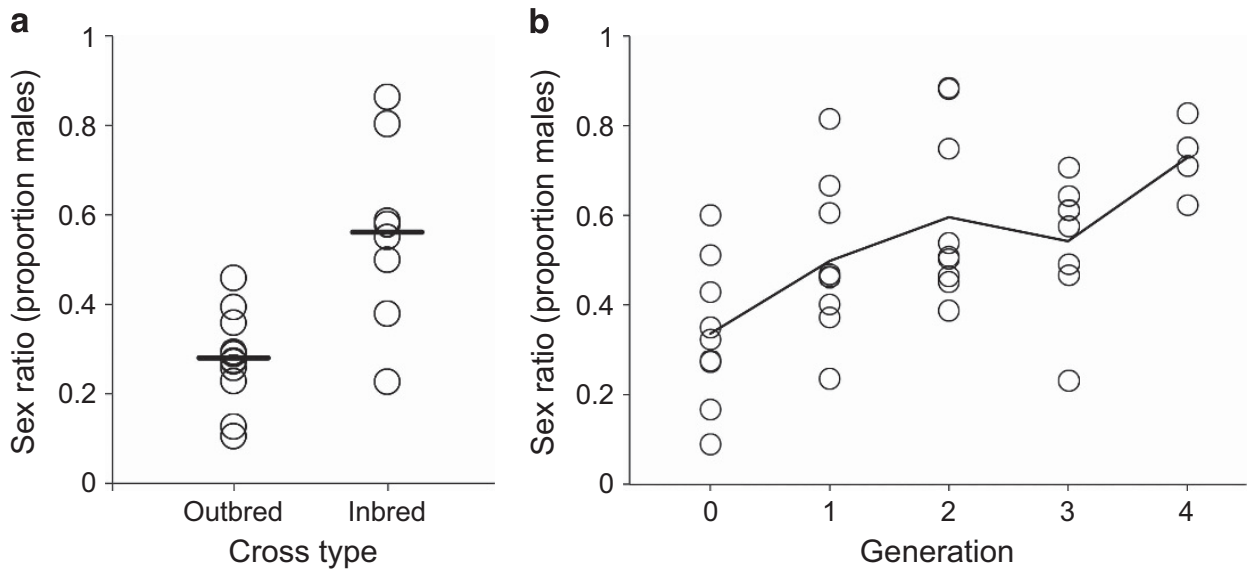

Figure 2 Change in sex ratio (proportion males) under inbreeding. (a) Each point represents the sex ratio (estimated from cocoons) of the progeny derived from a single mated pair, and horizontal bars indicate mean sex ratios. Compared with outbred families (RB126 female $\times$ RB093 male, $n=13$ ), inbred families (RB126 female $\times$ RB126 male, $n=8$ ) have a higher sex ratio. (b) Each point represents the sex ratio from an individual lab line (estimated from adult emergence data) for a given generation in the lab (generations $0-2, n=9$; generation 3, $n=8$; generation 4, $n=4$ ). The line tracks mean sex ratio over five generations of lab rearing. As predicted under CSD, inbreeding in the lab results in an increase in secondary sex ratio. In both (a) and (b), sex ratios are untransformed.

sl-CSD or a special case of ml-CSD in which only one sex-determining locus was heterozygous in the mother.

The impact of inbreeding on sex ratio was evident in both individual families and in propagation lines. Consistent with CSD, inbred families had significantly higher sex ratios than outbred families ( $t$-tests: cocoon sex ratio $t_{9.20}=3.55, P=0.0030$; adult sex ratio $t_{15.78}=3.05, P=0.0038$; log-binomial regressions: cocoon sex ratio $Z=3.93, P<0.0001$; adult sex ratio $Z=3.14, P=0.0009$; Figure 2a). Cocoon sex ratio and adult sex ratio were significantly correlated (inbred families: Pearson's $r=0.88, P=0.0039$; outbred families: $r=0.78, P=0.0019$ ), indicating that cocoon weight is a reliable indicator of adult sex. Examination of the sex ratio in individual inbred families revealed that five out of eight families had more males than expected based on observed sex ratios in outbred families (mean: 0.28 ; $95 \%$ confidence interval: $0.22-0.34$; Figure 3 ). Of these five, two families had extremely male-biased sex ratios. As discussed below, we suspect that these families were derived from diploid male fathers.

Going a step further, we next asked whether the sex ratios in our three putatively matched families are consistent with expectations under sl-CSD and ml-CSD. Under CSD, and assuming a mated pair shares an allele at every sex-determining locus, the expected proportion of diploid males among the fertilized offspring is $0.5^{h}$, where $h$ is the number of heterozygous sex-determining loci in the female. Assuming diploid male survival (DMS) is equal to that of other 
offspring, the expected sex ratio $(r)$ arising from a matched mating is: $(1-f)+0.5^{h} f$, where $f$ is the fertilization rate. If survival of diploid males is reduced relative to other offspring, $r=\left[(1-f)+\left(0.5^{h} f \delta\right)\right] /$ $\left[(1-f)+\left(0.5^{h} f \delta\right)+\left(1-0.5^{h}\right) f\right]$, where $\delta=$ DMS (Cook, 1993b; Heimpel et al., 1999; Wu et al., 2005; de Boer et al., 2008). Under this model, certain combinations of sex ratio, developmental mortality and DMS are not possible (Cook, 1993b). The permissible combinations of sex ratio and mortality across a range of DMS rates are depicted in Figure 4. The observed range of sex ratios and mortality rates (calculated for each family as the proportion of eggs that did not survive to the cocoon stage) for our matched families indicate that our data are consistent with a single heterozygous sex-determining locus, so long as DMS is at least $50 \%$ and consistent with ml-CSD under all levels of DMS.

Next, we used our estimated fertilization rates (mean: 0.72; 95\% confidence interval: $0.66-0.78$; derived from the observed sex ratios in outbred families) to more fully explore the levels of DMS that are consistent with our data. Assuming a single heterozygous sexdetermining locus, our observed sex ratios are consistent with expected sex ratios so long as DMS is at least 50\% (Figure 4), but no more than $89 \%$ (Figure 5a). In contrast, if we assume two heterozygous sex-determining loci, there is no level of DMS that could produce our observed sex ratios, given our observed fertilization rates (Figure 5b). Together, these analyses imply that there was a single variable sex-determining locus and a DMS value between 50 and $89 \%$.

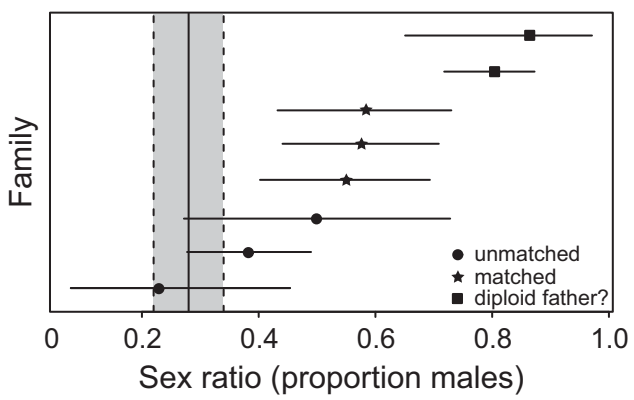

Figure 3 Comparison of inbred family sex ratios to unmatched expectations. Horizontal lines depict mean sex ratios and $95 \%$ confidence intervals for each inbred family. Unmatched expectations are based on observed sex ratios in outbred families (solid vertical line: mean sex ratio; gray area: 95\% confidence interval). Five families have sex ratios that fall outside of these expectations. Extreme male bias in two families may be the product of diploid male fathers (see Discussion).

Figure 4 Comparison of observed sex ratios and developmental mortality rates to permissible values under CSD and five levels of DMS. In each plot, the data point is the mean sex ratio and mortality rate (egg to cocoon) for the three putatively 'matched' inbred families (Figure 3); error bars reflect the range of observed values. Sex ratios $(r)$ were calculated for all possible fertilization rates $(f)$ as: $r=\left[(1-f)+\left(0.5^{h} f \delta\right)\right] /\left[(1-f)+\left(0.5^{h} f \delta\right)+\left(1-0.5^{h}\right) f\right]$, where $\delta=$ DMS and $h=$ the number of sex-determining loci at which the mother was heterozygous. The minimum developmental mortality $(\mu)$ consistent with CSD was calculated as $\mu=(1-\delta) f / 2^{h}$. Data points outside of the light gray area are not consistent with a single heterozygous sexdetermining locus; data points outside of the light and dark gray area are not consistent with either one or two heterozygous sex-determining loci. These figures indicate that our data are consistent with ml-CSD under all levels of DMS and sl-CSD, so long as DMS is $>50 \%$.
Similar to the ploidy data, these results are consistent with either slCSD or a special case of ml-CSD in which variation had been lost at all but one locus. Notably, the observed sex ratios for our two malebiased families ( $r=0.80$ and 0.86$)$ fall well outside of expectations for both sl-CSD and ml-CSD (Figure 5). We therefore suspect that these two families were fathered by diploid males (see Discussion).
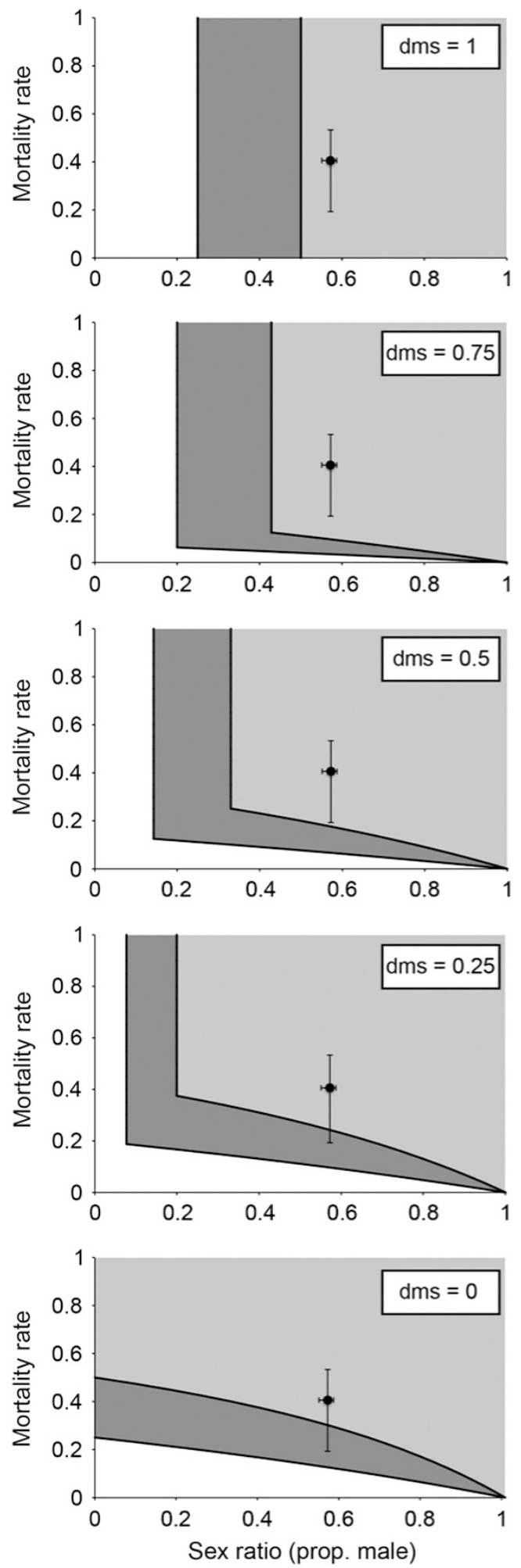
Analysis of multi-line multi-generational rearing data provides additional support for CSD. We found that sex ratio was positively correlated with the number of generations of inbreeding in the lab (repeated measures analysis of variance: $\mathrm{F}_{4,25.9}=4.95, P=0.0042$; binomial generalized linear model: $F_{4,26}=13.94, \quad P<0.0001$; Figure $2 \mathrm{~b}$ ). The post hoc tests reveal that this increase in sex ratio occurred rapidly. For example, by G1, there was a significantly higher sex ratio than G0 (repeated measures analysis of variance ANOVA: $t_{21}=-2.14, P=0.022$, Supplementary Table S2; binomial generalized linear model: $\mathrm{t}_{26}=-2.54, P=0.0088$, Supplementary Table S3). Following G1, we did not see any significant changes in sex ratio until G4, at which point there was a sudden increase in sex ratio, followed by widespread population crashes (Figure $2 \mathrm{~b}$ and Supplementary Tables S2 and S3). Qualitatively, these patterns resemble expectations under sl-CSD, in which inbreeding leads to a rapid increase in sex ratio, followed by population crashes stemming from diploid male production. In contrast, ml-CSD generally requires many generations of inbreeding to produce a detectable shift in sex ratio (Cook, 1993b; de Boer et al., 2008).

\section{Assessment of inbreeding depression}

Of 20 outbred pairs that mated, 14 females laid eggs, 13 of which produced viable larvae. Of 13 inbred pairs that mated, 11 females laid eggs, 11 of which produced viable larvae. Inbred and outbred pairs did not differ significantly in the proportion of females that laid eggs (one-tailed Fisher's exact test, $P=0.92$ ) or in the proportion of females that failed to produce viable larvae (one-tailed Fisher's exact test, $P=1.00)$. There was also no difference in the number of eggs laid per female $\left(t_{19.57}=-0.52 ; P=0.30\right.$; Table 1$)$. Inbred and outbred families did differ, however, in the egg to cocoon survival rate $\left(t_{18.752}=-2.04\right.$; $P=0.028$ ). Examination of stage-specific numbers and survival rates indicates that much of this difference stems from differential survival during the larval period (Table 1).

To gain additional insight into diploid male survival, we compared the numbers of male and female progeny produced by inbred and outbred families. Under CSD, some proportion of diploid progeny from inbred families will develop as diploid males. If there is no reduction in diploid male survival, we expect significantly fewer females and significantly more males in inbred families. If diploid male survival is low, however, we would expect to see a reduction in the number of females without a corresponding increase in the number of males. We found that whereas inbred families produced significantly fewer females than outbred families (adults: $t_{19}=-2.91$, $P=0.0089$; cocoons: $t_{19}=-4.94, P<0.0001$ ), there was no difference in the number of males (adults: $t_{19}=0.20, P=0.84$; cocoons: $t_{19}=0.17, P=0.87$; results for the full model and post hoc tests can be found in Supplementary Tables S4 and S5). These results indicate that the increased sex ratio in inbred families is because of a loss of
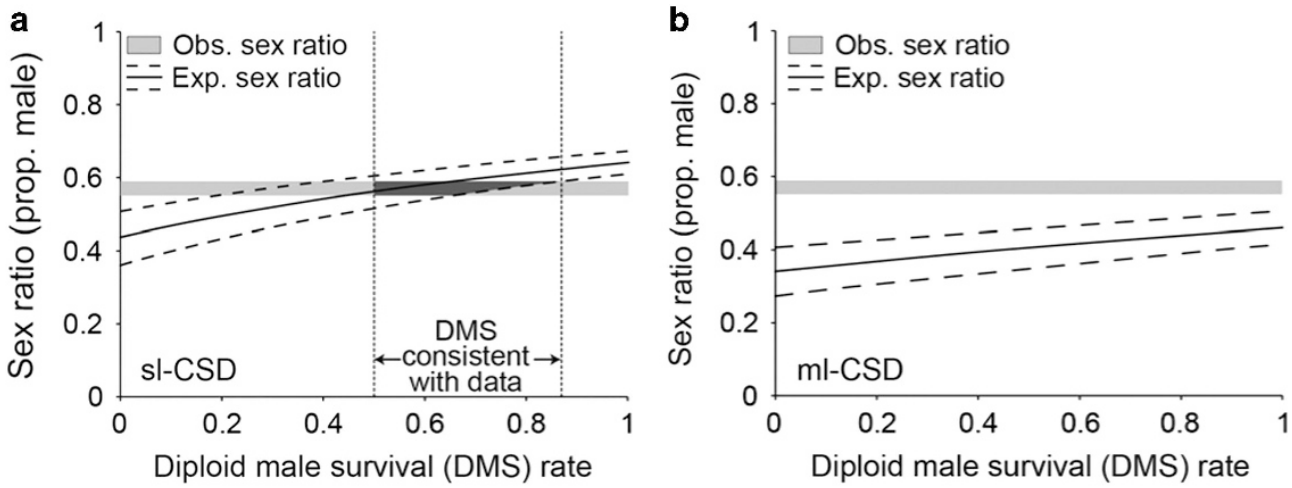

Figure 5 Comparison of observed sex ratios to expected ratios calculated as a function of DMS under sl-CSD and ml-CSD. Expected sex ratios were calculated using the $95 \%$ confidence interval for fertilization rate estimated from outbred families, assuming a single heterozygous sex-determining locus (a) and two heterozygous sex-determining loci (b). Light gray bars indicate the range of sex ratios observed in three putatively 'matched' inbred families (Figure 3). Dark gray shading indicates areas where observed and expected values overlap. Observed sex ratios are consistent with a single heterozygous sexdetermining locus so long as DMS is $<89 \%$ (minimum value of $50 \%$ is inferred on the basis of Figure 4 ). Under the two-locus model, there is no overlap between observed and expected sex ratios.

Table 1 Rearing and survival outcomes (mean \pm s.e.m.) for progeny of outbred (RB126 $\times$ RB093) and inbred (RB126 $\times$ RB126) families

\begin{tabular}{|c|c|c|c|c|}
\hline & Outbred $(n=14)$ & Inbred $(\mathrm{n}=11)$ & Test statistic $\left(\mathrm{t}_{\text {d.f. }}\right)$ & $\mathrm{P}$-value $\mathrm{a}^{\mathrm{a}}$ \\
\hline No. of eggs & $118.1 \pm 9.9$ & $108.8 \pm 15.1$ & $t_{19.6}=-0.52$ & 0.3043 \\
\hline No. of larvae & $104.5 \pm 11.0$ & $93.2 \pm 13.2$ & $t_{22.0}=-0.36$ & 0.3623 \\
\hline No. of cocoons ${ }^{b}$ & $86.0 \pm 10.2$ & $38.0 \pm 11.0$ & $t_{19.8}=-2.72$ & 0.0066 \\
\hline No. of adults & $54.6 \pm 9.9$ & $25.5 \pm 8.7$ & $t_{20.9}=-2.20$ & 0.0197 \\
\hline Egg hatching rate $(\%)^{\mathrm{b}}$ & $82.2 \pm 7.1$ & $85.2 \pm 2.7$ & $t_{17.2}=0.46$ & 0.6754 \\
\hline Larval cocooning rate $(\%)^{c}$ & $79.9 \pm 5.5$ & $49.6 \pm 11.5$ & $t_{13.3}=-2.17$ & 0.0244 \\
\hline Egg to cocoon survival (\%) & $67.7 \pm 6.9$ & $42.3 \pm 9.3$ & $t_{18.8}=-2.04$ & 0.0280 \\
\hline Cocoon emergence rate $(\%)^{d}$ & $60.2 \pm 7.4$ & $57.2 \pm 10.0$ & $t_{14.7}=-0.40$ & 0.3471 \\
\hline
\end{tabular}

aAll $P$-values are derived from one-tailed $t$-tests performed on transformed data (untransformed averages and s.e.m. are reported). Bold values are significant at $\alpha=0.05$.

bexcludes one family with missing larval data (outbred $n=13$ and inbred $n=11$ ).

cExcludes one family with missing larval data and one family that failed to hatch (outbred $n=12$ and inbred $n=11$ ).

dExcludes three families that failed to produce cocoons (outbred $n=13$ and inbred $n=9$ ). 
females without a concomitant increase in males, suggesting that an appreciable number of diploid males are dying during development.

Beyond survival, we also compared female fecundity in inbred and outbred families. Examination of the relationship between female weight and egg number indicates that the two are strongly correlated (Pearson's $r=0.85, P<0.0001$; Figure 6). Thus, cocoon weight is a good predictor of female fecundity. When we compared female cocoon weight between inbred and outbred lines, however, we found no difference after controlling for family-level variation $\left(t_{7}=0.12\right.$, $P=0.45)$. In contrast, inbred males were significantly heavier than outbred males $\left(t_{8}=1.92, P=0.046\right)$. Given that the haploid males should not differ between inbred and outbred families (all haploid males are the progeny of RB126 females), this finding is most likely because of the presence of heavy diploid male cocoons in the inbred families.

\section{Assessment of inbreeding avoidance}

In our first set of no-choice mating assays, 20 out of 21 outbred pairs and 13 out of 18 inbred pairs mated (Figure 7). Although a smaller proportion of inbred pairs mated than outbred pairs, this difference was marginally nonsignificant $(P=0.061$; one-tailed Fisher's exact

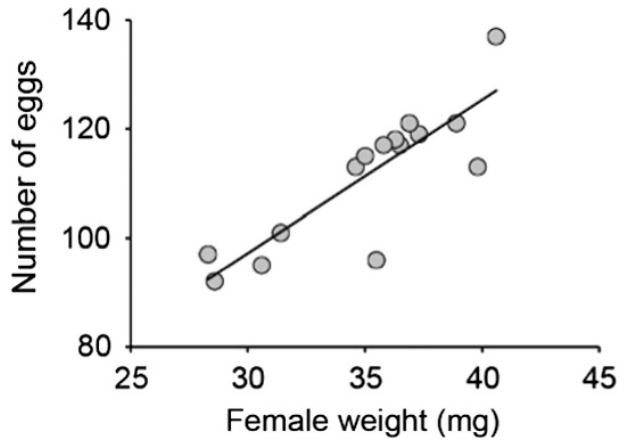

Figure 6 Relationship between cocoon weight and female fecundity. Individual females were weighed and then dissected to determine egg number. Female weight is positively correlated with egg number $(n=15$; Pearson's $r=0.85 ; P<0.0001$ ), indicating that weight is a good predictor of fecundity in $N$. lecontei.

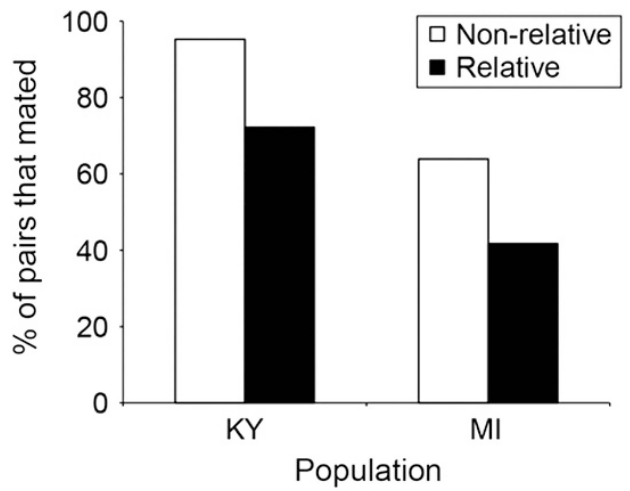

Figure 7 Mating success for related and unrelated pairs, by population. Females from KY that were paired with males from the same line tended to mate less than females paired with males from a different line ( $n=18$ same line; $n=21$ different line; $P=0.061$ ). Sibling pairs from $\mathrm{MI}$ were less willing to mate than nonsibling pairs ( $n=36$ sibling pairs; $n=36$ nonsibling pairs; $P=0.048$ ). Together, these results are consistent with inbreeding avoidance in $N$. lecontei. test). In our second set of no-choice assays, 23 out of 36 nonsibling pairs mated and 15 out of 36 sibling pairs mated (Figure 7). Consistent with inbreeding avoidance, sibling pairs were significantly less willing to mate than nonsibling pairs $(P=0.048$; one-tailed Fisher's exact test). Of the pairs that did mate, we did not observe any differences in the latency time to mating $\left(\mathrm{F}_{1,31}=0.30, P=0.59\right.$ for inbred/outbred assays; $\mathrm{F}_{1,36}=0.74, P=0.39$ for sibling/nonsibling assays).

\section{DISCUSSION}

To date, sex determination has been investigated in many hymenopteran taxa, but species from the suborder Symphyta are notably underrepresented (van Wilgenburg et al., 2006). Moreover, there are relatively few taxa for which sex determination has been investigated between closely related species, making it difficult to draw conclusions about the lability of this trait and correlated evolution with other traits (notable exceptions include the genera Cotesia, Diadegma and Habrobracon; reviewed in van Wilgenburg et al., 2006 and Harpur et al., 2013). Our study addresses these gaps and confirms that $N$. lecontei, like its congener N. nigroscutum (Wallace and Smith, 1971), has CSD. Although we have not definitively ruled out ml-CSD, our data are also consistent with sl-CSD. In addition, via investigation of inbreeding depression and inbreeding avoidance, we gain further insight into how this sex determination mechanism may be shaping evolution in this species.

In terms of sex determination mechanism, our data confirm two key predictions of CSD in N. lecontei: that inbreeding will result in the production of diploid males (Figure 1) and increased sex ratios (Figure 2). In addition, the following observations suggest that sex is most likely determined by a single locus in this species: (1) the ploidy analysis detected more 'matched' sibling pairs than were expected under ml-CSD; (2) observed sex ratios in our inbred families could not be explained by ml-CSD (Figure 5); and (3) sex ratio in laboratory lines reared en masse increased more rapidly than might be expected under ml-CSD (Figure 2b). An important limitation of these data is that our rearing design does not allow us to rule out the possibility that, through loss of heterozygosity at sex-determining loci, ml-CSD 'collapsed' into sl-CSD in our lab lines (de Boer et al., 2008; Heimpel and de Boer, 2008). However, observation of patterns consistent with sl-CSD in multiple independent lab lines renders this alternative explanation less likely. Moreover, a recent population genomic analysis of this species revealed that diploid males are produced at detectable levels in natural populations (two out of the six adult males included in the analysis were diploid; R Bagley, personal observation) - this observation is more likely under sl-CSD than ml-CSD. Nevertheless, although evidence pointing to sl-CSD is strong in this species, definitive proof of this sex determination mechanism will require additional crosses that control for the allelic composition of sibling parents and verify the ploidy of male offspring.

In terms of inbreeding depression, our survival data indicate that inbred families experience higher mortality than outbred families over the course of larval development (Table 1). Two lines of evidence indicate that this mortality stems, at least in part, from the death of diploid males. First, for the three putatively 'matched' families, the maximum DMS that is consistent with our observed sex ratio is $89 \%$ (Figure 5a). Second, the fact that we observe significantly fewer diploid females in inbred families without a corresponding increase in the number of males implies that diploid males are dying during development. In contrast, diploid males and females have equal viability in most other taxa studied to date, including N. nigroscutum (Wallace and Smith, 1971; Harpur et al., 2013). 
In contrast to the three inbred families with moderately male-biased sex ratios, two inbred families have male-biased sex ratios that far exceeded expectations under sl-CSD or ml-CSD (Figure 5). One possible explanation for these unusually high sex ratios is that they reflect among-family variation in fertilization rate and mortality. Alternatively, the two families with markedly male-biased sex ratios may have been produced by diploid male fathers. This explanation is consistent with the observation that, in all haplodiploid species examined to date, females mated to diploid males produce more male-biased sex ratios than do females mated to matched haploid males (Harpur et al., 2013). For example, in N. nigroscutum, a mating between a female and a diploid male resulted in progeny that were $89 \%$ male, whereas matings between females and matched haploid males produced progeny that were $69 \%$ male (Wallace and Smith, 1971). Cytological studies confirmed that $N$. nigroscutum produces unreduced diploid sperm and that a female mated to a diploid male produced many haploid male sons and a handful of triploid daughters that were found to be highly sterile (Wallace and Smith, 1971). Similarly, inviable or sterile triploid daughters have been reported in over two-thirds of studies that have examined diploid male fertility (Harpur et al., 2013). Although it is reasonable to speculate that diploid $N$. lecontei males would produce similar results, fertile diploid males that produce viable diploid daughters have been reported in two species (Cowan and Stahlhut, 2004; Elias et al., 2009), one of which has a congener that produces sterile triploid females (de Boer et al., 2007). Thus, additional studies are needed to evaluate the mating ability and fertility of diploid N. lecontei males.

Although the fertility of diploid $N$. lecontei males remains an open question, our data suggest that diploid males generally have reduced viability. Thus, the production of partially inviable, and possibly infertile, diploid males represents one source of inbreeding depression in $N$. lecontei. In contrast, our data do not reveal any obvious inbreeding depression stemming from reduced fertility of diploid females. Although inbreeding depression unrelated to DMP should be minimal in haplodiploid taxa because of the purging of recessive alleles, alleles with female-specific or overdominant effects may still reduce fitness in inbred females (Bruckner, 1978; Henter, 2003). Consistent with this prediction, multiple studies have reported reduced viability and/or fertility of inbred females in haplodiploid taxa (Bruckner, 1978; Henter, 2003; Schrempf et al., 2006). Although a lack of alleles with sex-limited or overdominant effects is one explanation for our lack of inbreeding depression in N. lecontei females, another explanation is that these families were not inbred enough-and heterozyosity levels not sufficiently reduced-to produce detectable inbreeding depression. Assuming that the founding members of the RB126 colony had an inbreeding coefficient (F) of zero and that the inbred females we examined were produced via two generations of brother-sister matings, the expected value of $\mathrm{F}$ for these females is $\sim 0.375$. In contrast, most other studies that have documented inbreeding depression in haplodiploid females did so after three or more generations of inbreeding (see, for example, Bruckner, 1978; Henter, 2003; Schrempf et al., 2006; in all cases F $>0.50$ ). Thus, additional data are needed to determine whether female-specific and overdominant alleles contribute to inbreeding depression in this species. We can conclude, however, that inbreeding depression via DMP - which requires only a single generation of sibmating - has a more immediate impact on $N$. lecontei fitness than inbreeding depression in females.

Given our finding that $N$. lecontei experiences inbreeding depression, natural selection is expected to favor traits that reduce the probability of inbreeding in this species. In many taxa, temporal or spatial segregation of the sexes obviates the need for additional behavioral inbreeding avoidance mechanisms (Pusey and Wolf, 1996). Like the males of many Neodiprion species, N. lecontei males have one less feeding instar than females and therefore tend to leave the trees to spin cocoons earlier (Wilson et al., 1992). Nevertheless, in every single family for which we tracked adult emergence, at least some male and female siblings emerged on the very same day. Because males and females are able to mate shortly after emergence (Benjamin, 1955; Wilson et al., 1992), same-day emergence should translate directly into mating opportunity (but see Ode et al., 1995). In addition, even when males and females do not emerge on the exact same day, they may still do so within the average lifespan of their siblings (4.5 days for females and 3.2 days for males; Wilson et al., 1992).

In addition to temporal emergence patterns, it is also important to consider how siblings are distributed spatially: if siblings are spread out across the environment, encounters between siblings might be too rare to generate selection for inbreeding avoidance. For this reason, inbreeding avoidance via kin recognition is expected to be more common in group-living animals than in solitary animals (Pusey and Wolf, 1996; van Wilgenburg et al., 2006; Lihoreau et al., 2007; but see Metzger et al., 2010). In N. lecontei, siblings start their life in close proximity because females of this species lay many eggs per needle and also tend to deposit their entire complement on a single branch tip (Benjamin, 1955; Craig and Mopper, 1993). Throughout development, $N$. lecontei larvae tend to remain tightly clustered until the final nonfeeding instar, which usually spins a cocoon in the soil beneath the host tree (Benjamin, 1955; Wilson et al., 1992). Upon emerging, adults typically return to the canopy to mate. Given their tendency to remain tightly clustered throughout the larval feeding period, it is likely that siblings would remain clustered to some degree as cocoons and, as a result, encounter one another as emerging adults.

Based on our rearing data and published accounts of $N$. lecontei natural history, we conclude that siblings have a nonnegligible probability of encountering one another as reproductive adults. Given our observation that inbreeding depression occurs in this species, natural selection should favor individuals that avoid mating with kin. Consistent with these predictions, relatives appear less willing to mate than nonrelatives (Figure 7). To our knowledge, this is the first documentation of behaviorally based inbreeding avoidance in a Symphytan lineage, thus adding to a growing list of insects across diverse taxa with similar behaviors (Pusey and Wolf, 1996; ShellmanReeve, 2001; Lihoreau et al., 2007; Metzger et al., 2010). However, we have not yet identified the proximate mechanism underlying kin recognition in this species. Because relatives were always reared together in our experiments, we could not determine whether kinrecognition cues are derived from a shared rearing environment, learned from prior association with conspecifics or learned from an individual's own phenotype (Pusey and Wolf, 1996). Intriguingly, at least one parasitic wasp species appears to use the CSD locus itself to avoid genetically incompatible (that is, matched) mates, regardless of their relatedness (Thiel et al., 2013). Beyond identifying the cues used for kin recognition in this species, additional work is also needed to determine whether $N$. nigroscutum, which also suffers from inbreeding depression stemming from sl-CSD (diploid males have drastically reduced copulation success and fertility; Wallace and Smith, 1971), also exhibits inbreeding avoidance. Unlike N. lecontei, N. nigroscutum siblings tend to be distributed across multiple host plants (Becker and Benjamin, 1967) and therefore less likely to encounter one another as reproductive adults. Thus, we would predict that kin discrimination 
would be weak or absent in this solitary species (but see Metzger et al., 2010).

CSD can also have important consequences for the probability of population extinction. Whereas in large populations, a large number of segregating CSD alleles can minimize the probability of DMP, genetic drift in small populations reduces the number of CSD alleles, making DMP — even between unrelated individuals - more likely (Cook and Crozier, 1995; Zayed and Packer, 2005). Using stochastic simulations, Zayed and Packer (2005) demonstrated that DMP can lead to an 'extinction vortex' in bottlenecked populations, rendering haplodiploids with CSD especially prone to extinction. Consistent with these predictions, a frequent pattern we have observed in our laboratory lines is a sudden increase in the secondary sex ratio (presumably as the result of females refusing to mate or mating with infertile diploid males), followed by a crash (for example, Figure 2b). However, several traits can reduce the probability of entering an extinction vortex, including: high female fecundity, female-biased sex ratio, inbreeding avoidance and reduced viability (or mating ability) of diploid males (Hedrick et al., 2006; Hein et al., 2009). In this study, we found that fecundity was high ( $>100$ eggs laid per female; Table 1), sex ratios were heavily female biased (Figure 2), sibling pairs were less likely to mate than nonsiblings (Figure 7) and diploid males had reduced viability (Figure 5). Thus, except under the most extreme conditions (for example, forced breeding of siblings in the lab), $N$. lecontei may be unlikely to enter a diploid male extinction vortex.

Like N. lecontei, many other diprionid species are characterized by high female fecundity (Larsson et al., 1993) and female-biased sex ratios (Craig and Mopper, 1993). As such, pine sawflies may be less prone to population extinction than haplodiploids lacking these features. These same traits may have also contributed to the success of invasive diprionids. For example, over the past century, four nonnative diprionid species have become established pests in North America: Neodiprion sertifer, Diprion similis, Gilpinia frutetorum and Gilpinia hercyniae. Of these, sex determination mode is known only for G. hercyniae, which is obligately thelotokous and therefore immune to DMP (Smith, 1941). Although thelotoky undoubtedly facilitated the establishment of G. hercynaie, additional work is needed to determine whether and how the other invasive diprionid species overcame the limitations of CSD in genetically depauperate founding populations (see, for example, Zayed et al., 2007).

To date, CSD, inbreeding depression and inbreeding avoidance have each been documented in a taxonomically diverse collection of hymenopteran species. However, although taxonomic breadth is important for making inferences regarding ancestral character states (see, for example, Asplen et al., 2009), disentangling the complex interplay between ecology, mating behavior and sex determination will require comparative studies between closely related populations and species (Heimpel and de Boer, 2008). The ideal study systems are those that vary in sex determination mode (for example, Cotesia) or in traits that are related-either directly or indirectly— to sex determination mode (for example, diploid male viability and fertility, traits influencing the spatial or temporal distribution of siblings and traits related to mate choice). For example, our finding that a gregarious Neodprion species has CSD and exhibits inbreeding avoidance sets the stage for future work examining sex determination and mate choice in additional Neodiprion species that vary from solitary to highly gregarious (Larsson et al., 1993). Additional priorities for future work on $N$. lecontei and other diprionid species include experimentally verifying sl-CSD, quantifying diploid male survival and fertility and elucidating the mechanisms underlying kin-based mating preferences.
Ultimately, these studies have the potential to help us better understand both the causes (molecular mechanisms) and consequences (susceptibility to inbreeding depression and population extinction) of haplodiploidy.

\section{DATA ARCHIVING}

All data (flow cytometry, sex ratio, survival, cocoon weights and mating data) are available from the Dryad Digital Repository: http://dx.doi.org/10.5061/dryad.ck3b6.

\section{CONFLICT OF INTEREST}

The authors declare no conflict of interest.

\section{ACKNOWLEDGEMENTS}

We are indebted to George Heimpel, members of the Linnen lab and three anonymous reviewers for helpful comments and/or discussions that improved previous versions of this manuscript. We thank Todd Rounsaville and the University of Kentucky Arboretum for permission to collect sawflies and for use of their orchard ladder. For advice on statistical analysis, we thank Connie Wood, Heather Bush and Kristen McQuerry. We thank Kathryn Deveau for generating data used in this study and other members of the Linnen lab, especially Melanie Hurst and Adam Leonberger, for assistance with sawfly rearing and plant maintenance. Funding for this research was provided by the National Science Foundation (DEB-1257739; to CRL), a USDA-NIFA Predoctoral Fellowship (2015-67011-22803; to RKB) and University of Kentucky Multi-Year and Daniel R. Reedy fellowships (to RKB).

Asplen MK, Whitfield JB, de Boer JG, Heimpel GE (2009). Ancestral state reconstruction analysis of hymenopteran sex determination mechanisms. J Evol Biol 22: 1762-1769. Becker GC, Benjamin DM (1967). Biology of Neodiprion nigroscutum (Hymenoptera: Diprionidae) in Wisconsin. Can Entomol 99: 146-159.

Benjamin DM (1955). The biology and ecology of the red-headed pine sawfly. USDA Tech Bull 118: 55.

Beukeboom LW (1995). Sex determination in Hymenoptera: a need for genetic and molecular studies. Bioessays 17: 813-817.

Beukeboom LW, Kamping A, van de Zande $L$ (2007). Sex determination in the haplodiploid wasp Nasonia vitripennis (Hymenoptera: Chalcidoidea): a critical consideration of models and evidence. Semin Cell Dev Biol 18: 371-378.

Bruckner D (1978). Why are there inbreeding effects in haplo-diploid systems? Evolution 32: $456-458$.

Cook JM (1993a). Sex determination in the Hymenoptera: a review of models and evidence. Heredity 71: 421-435.

Cook JM (1993b). Experimental tests of sex determination in Goniozus nephantidis (Hymenoptera: Bethylidae). Heredity 71: 130-137.

Cook JM, Crozier RH (1995). Sex determination and population biology in the Hymenoptera. Trends Ecol Evol 10: 281-286.

Cowan DP, Stahlhut JK (2004). Functionally reproductive diploid and haploid males in an inbreeding hymenopteran with complementary sex determination. Proc Natl Acad Sci USA 101: 10374-10379.

Craig TP, Mopper S (1993). Sex ratio variation in sawflies. In: Wagner MR, Raffa KF (eds). Sawfly Life History Adaptations to Woody Plants. Academic Press: San Diego. pp 61-93.

Crozier RH (1971). Heterozygosity and sex determination in haplo-diploidy. Am Nat 105: $399-412$.

Cunha AB, Kerr WE (1957). A genetical theory to explain sex determination by arrhenotokous parthenogenesis. Forma Funct 1: 33-36.

de Boer JG, Ode PJ, Rendahl AK, Vet LEM, Whitfield JB, Heimpel GE (2008). Experimental support for multiple-locus complementary sex determination in the parasitoid Cotesia vestalis. Genetics 180: 1525-1535.

de Boer JG, Ode PJ, Vet LEM, Whitfield J, Heimpel GE (2007). Complementary sex determination in the parasitoid wasp Cotesia vestalis (C. plutellae). J Evol Biol 20: 340-348.

Dobson SL, Tanouye MA (1998). Evidence for a genomic imprinting sex determination mechanism in Nasonia vitripennis (Hymenoptera; Chalcidoidea). Genetics 149: 233-242.

Elias J, Mazzi D, Dorn S (2009). No need to discriminate? Reproductive diploid males in a parasitoid with complementary sex determination. PLOS ONE 4: e6024.

Godfray HCJ (1994). Parasitoids: Behavioral and Evolutionary Ecology. Princeton University Press: Princeton, NJ, USA.

Hare EE, Johnston JS (2011). Genome size determination using flow cytometry of propidium iodide-stained nuclei. In: Orgogozo V, Rockman MV (eds). Molecular Methods for Evolutionary Genetics, vol. 772. Humana Press: New York, NY, USA. pp 3-12. 
Harpur BA, Sobhani M, Zayed A (2013). A review of the consequences of complementary sex determination and diploid male production on mating failures in the Hymenoptera. Entomol Exp App/ 146: 156-164.

Hedrick P, Gadau J, Pagejr R (2006). Genetic sex determination and extinction. Trends Ecol Evol 21: 55-57.

Heimpel GE, Antolin MF, Strand MR (1999). Diversity of sex-determining alleles in Bracon hebetor. Heredity 82: 282-291.

Heimpel GE, de Boer JG (2008). Sex determination in the Hymenoptera. Annu Rev Entomol 53: 209-230.

Hein S, Poethke H-J, Dorn S (2009). What stops the 'diploid male vortex'?-A simulation study for species with single locus complementary sex determination. Ecol Model 220: 1663-1669.

Henter HJ (2003). Inbreeding depression and haplodiploidy: experimental measures in a parasitoid and comparisons across diploid and haplodiploid insect taxa. Evolution 57: 1793-1803.

Larsson S, Bjorkman C, Kidd NAC (1993). Outbreaks in diprionid sawflies: why some species and not others? In: Wagner M, Raffa KF (eds). Sawfly Life History Adaptations to Woody Plants. Academic Press: San Diego. pp 453-479.

Lee JM, Hashino Y, Hatakeyama M, Oishi K, Naito T (1998). Egg deposition behavior in the haplodiploid sawfly Athalia rosae ruficornis Jakovlev (Hymenoptera: Symphyta: Tenthredinidae). J Insect Behav 11: 419-428.

Lihoreau M, Zimmer C, Rivault C (2007). Kin recognition and incest avoidance in a group living insect. Behav Ecol 18: 880-887.

Metzger M, Bernstein C, Hoffmeister TS, Desouhant E (2010). Does kin recognition and sib-mating avoidance limit the risk of genetic incompatibility in a parasitic wasp? PLoS ONE 5: e13505.

Niyibigira El, Overholt WA, Stouthamer R (2004). Cotesia flavipes Cameron and Cotesia sesamiae (Cameron) (Hymenoptera: Braconidae) do not exhibit complementary sex determination: evidence from field populations. Appl Entomol Zool 39: 705-715.

Ode PJ, Antolin MF, Strand MR (1995). Brood-mate avoidance in the parasitic wasp Bracon hebetor Say. Anim Behav 49: 1239-1248.

Poirie M, Periquet G, Beukeboom LW (1992). The hymenopteran way of determining sex. Semin Dev Biol 3: 357-361.

Pusey A, Wolf M (1996). Inbreeding avoidance in animals. Trends Ecol Evol 5347: 298-301.
Ruf D, Mazzi D, Dorn S (2010). No kin discrimination in female mate choice of a parasitoid with complementary sex determination. Behav Ecol 21: 1301-1307.

Schrempf A, Aron S, Heinze J (2006). Sex determination and inbreeding depression in an ant with regular sib-mating. Heredity 97: 75-80.

Shellman-Reeve JS (2001). Genetic relatedness and partner preference in a monogamous, wood-dwelling termite. Anim Behav 61: 869-876.

Smith SG (1941). A new form of spruce sawfly identified by means of its cytology and parthenogenesis. Sci Agric 21: 245-305.

Snell BGD (1935). The determination of sex in Habrobarcon. Genetics 21: 446-453.

Stouthamer R, Luck RF, Werren JH (1992). Genetics of sex determination and the improvement of biological control using parasitoids. Environ Entomol 21: 427-435.

Thiel A, Weeda AC, de Boer JG, Hoffmeister TS (2013). Genetic incompatibility drives mate choice in a parasitic wasp. Front Zool 10: 43.

van de Zande L, Verhulst EC (2014). Genomic imprinting and maternal effect genes in haplodiploid sex determination. Sex Dev 8: 74-82.

van Wilgenburg E, Driessen G, Beukeboom LW (2006). Single locus complementary sex determination in Hymenoptera: an 'unintelligent' design? Front Zool 3: 1.

Verhulst EC, Beukeboom LW, van de Zande $L$ (2010). Maternal control of haplodiploid sex determination in the wasp Nasonia. Science 328: 620-623.

Wallace DR, Smith SG (1971). Allelic sex determination in a lower hymenopteran, Neodiprion nigroscutum Midd. Can J Genet Cytol 13: 617-621.

Whiting PW (1943). Multiple alleles in complementary sex determination of Habrobracon. Genetics 28: 365-382.

Wilson LF, Wilkinson RC, Averill RC (1992). Redheaded Pine Sawfly: Its Ecology and Management. USDA Forest Service, Agriculture Handbook 694. USDA: Washington, DC.

Wu Z, Hopper KR, Ode PJ, Fuester RW, Tuda M, Heimpel GE (2005). Single-locus complementary sex determination absent in Heterospilus prosopidis (Hymenoptera: Braconidae). Heredity 95: 228-234.

Zayed A, Constantin SA, Packer L (2007). Successful biological invasion despite a severe genetic load. PLOS ONE 2: e868.

Zayed A, Packer L (2005). Complementary sex determination substantially increases extinction proneness of haplodiploid populations. Proc Natl Acad Sci USA 102: $10742-10746$

Zhou Y, Gu H, Dorn S (2006). Single-locus sex determination in the parasitoid wasp Cotesia glomerata (Hymenoptera: Braconidae). Heredity 96: 487-492.

Supplementary Information accompanies this paper on Heredity website (http://www.nature.com/hdy) 